\title{
A pilot randomised controlled trial of peripheral fractional oxygen extraction to guide blood transfusions in preterm infants
}

\author{
S P Wardle, R Garr, C W Yoxall, A M Weindling
}

Arch Dis Child Fetal Neonatal Ed 2002;86:F22-F27

See end of article for authors' affiliations

.....................

Correspondence to: Dr Wardle, Neonatal Unit, Queen's Medical Centre, University Hospital, Nottingham NG7 2UH, UK; steve.wardle@ mail.qmcuh-tr.trent.nhs.uk

Accepted

11 September 2001
Background: Peripheral fractional oxygen extraction (FOE) may be a better indicator of the need for transfusion than the haemoglobin concentration $(\mathrm{Hb})$ because it is a measure of the adequacy of oxygen delivery to meet demand. A randomised controlled trial of the use of peripheral FOE to guide the need for blood transfusions in preterm infants was carried out to test this hypothesis.

Method: Infants less than $1500 \mathrm{~g}$ birth weight who were stable and less than 2 weeks old were randomised to receive transfusions guided by either a conventional protocol based on $\mathrm{Hb}$ (conventional group) or a protocol based on measurements of peripheral FOE made by near infrared spectroscopy (NIRS group). Measurements of $\mathrm{Hb}$ and FOE were made on all infants from randomisation until discharge. The primary outcome measures were number of transfusions received, rate of weight gain, and postmenstrual age at discharge.

Results: Thirty seven infants were randomised to each group. Birth weight (median, range) (1200, 1004-1373 v 1136, 1009-1285 g) and $\mathrm{Hb}$ (median, range) at randomisation $(160,149-179 \mathrm{v}$ $155,145-181 \mathrm{~g} / \mathrm{l})$ did not differ between the two groups. The total number of transfusions given to the NIRS group was 56 and to the conventional group 84 . The median number of transfusions per infant, the median volume of blood transfused to each group, and the total number of donors to which infants were exposed were similar in the two groups. Infants transfused according to the conventional protocol were more likely to be transfused earlier and at a higher $\mathrm{Hb}$ than those transfused in the NIRS group. Infants in the conventional group spent a significantly shorter period than those in the NIRS group with $\mathrm{Hb}<100 \mathrm{~g} / \mathrm{l}$. Of the 56 transfusions given to the NIRS group, $33(59 \%)$ were given because of clinical concerns rather than because of high FOE. There was no difference in the rate of weight gain, rate of linear growth, postmenstrual age at discharge, or the incidence of chronic lung disease or retinopathy of prematurity.

Conclusions: FOE measurements failed to identify many infants felt by clinicians to require blood transfusion. This may have been because clinicians relied on conventional indicators of transfusion that are vague and non-specific, or a peripheral FOE of 0.47 alone may not be a sensitive enough predictor of the need for transfusion. This requires further study.
$\mathrm{H}$ aemoglobin concentration $(\mathrm{Hb})$ is generally kept higher in sick infants than in older or less intensively treated infants despite no clear evidence that this is beneficial. ${ }^{12}$ There are, however, suggestions that $\mathrm{Hb}$ may be a relatively poor indicator of tissue oxygenation. ${ }^{3-5}$ An alternative is peripheral fractional oxygen extraction (FOE), an indicator that reflects the adequacy of oxygen delivery to meet demand and is therefore likely to be a better marker of the need for transfusion than $\mathrm{Hb}$ alone. ${ }^{4} 67$

FOE is a measure of the balance between oxygen delivery and oxygen consumption. It can be calculated if the arterial and venous saturation of a tissue is known. ${ }^{8}$ FOE measurements for the whole body require knowledge of central (pulmonary) venous saturation, and this is not practical for small infants. However, measurements of FOE within specific organs or tissues can be made. Peripheral FOE can be measured non-invasively by near infrared spectroscopy (NIRS) with partial venous occlusion applied to the forearm. ${ }^{9}{ }^{10}$ Studies using this method showed that preterm infants with symptomatic anaemia had a higher mean peripheral FOE than controls, whereas preterm infants with asymptomatic anaemia were similar to controls. ${ }^{7}$ Peripheral FOE correlates positively with the haemoglobin $\mathrm{F}(\mathrm{HbF})$ fraction and negatively with the red cell volume, ${ }^{7}$ both important measures of the adequacy of the availability of oxygen to the tissues. ${ }^{311} 12$ These relations support the view that FOE is a good reflection of oxygen availability to the tissues.

Although transfusions are an important part of neonatal intensive care, there is a paucity of evidence to guide their effective use. Strategies for blood transfusions for preterm infants are based on consensus opinion rather than scientific evidence. ${ }^{2}$ Overtransfusion is potentially harmful and undertransfusion may also be detrimental. A precise approach to determining exactly when transfusions should be given is therefore very important.

The aim of this study was to test the hypothesis that measurements of FOE can be used to guide the need for blood transfusions. Our primary hypothesis was that infants assessed for the need for transfusion by repeated FOE measurements would receive fewer transfusions than those assessed in the conventional way using $\mathrm{Hb}$. We also hypothesised that a decrease in the number of blood transfusions given using this new monitoring method would not be harmful-that is, result in poorer growth, longer hospital stay,

Abbreviations: $\mathrm{FOE}$, fractional oxygen extraction; NIRS, near infrared spectroscopy 
or other clinical complications-and that fewer blood transfusions may be beneficial by reducing the incidence of retinopathy of prematurity and chronic lung disease.

\section{METHOD}

This pilot study was an open randomised controlled trial of measurements of peripheral FOE to guide the need for blood transfusions compared with our current protocol based on $\mathrm{Hb}$.

\section{Study population}

Newborn preterm infants admitted to the neonatal intensive care unit at Liverpool Women's Hospital were considered eligible if they were inborn and inbooked. This allowed the infants to be monitored until their discharge from hospital.

\section{Entry criteria}

Infants were included if: (a) their birth weight was below 1500 g; (b) their blood pressure was stable without inotropic support; $(c)$ they were not ventilated or ventilated with inspired oxygen less than $40 \%$; $(d)$ they were less than 2 weeks of age. Infants were given blood transfusions before entry into the trial according to standard written guidelines. Once randomised, infants were only transfused according to the trial protocols.

\section{Ethical approval}

The local research ethics committee approved the study and informed written parental consent was obtained before randomisation.

The entry criteria were intended to ensure that the infants in the study group were not critically ill and were in a stable clinical condition so that blood transfusions could be withheld if indicated by the FOE measurements despite fulfilling conventional criteria for transfusion. It was felt that if infants were sick or unstable, withholding transfusions could be hazardous, despite the absence of evidence to support the currently used transfusion guidelines.

\section{Randomisation}

Randomisation was by computerised random number allocation in sealed opaque envelopes. The envelopes were kept in another hospital department and drawn by an independent person from a separate department after a telephone call when an eligible patient had been recruited. Infants were stratified by birth weight into two groups: less than $1000 \mathrm{~g}$ and $1000-1500 \mathrm{~g}$.

Infants were randomised to one of two groups. Group 1 (conventional group) received blood transfusions according to a conventional protocol based on one used in previous studies $^{13}$ and used on our unit to guide transfusions before the start of this study. It is based on measurements of $\mathrm{Hb}$ and symptoms of anaemia. Transfusions were only given to infants in this group according to these criteria. Group 2 (NIRS group) received blood transfusions according to a protocol based on measurements of forearm FOE made using NIRS. The normal range of peripheral FOE was $0.23-0.47$ and was determined from a previous study as the 5th-95th centile for healthy nonanaemic infants. ${ }^{7}$ In this group, blood transfusions were only given when the forearm FOE was $\geqslant 0.47$, or if the clinicians responsible for the patient felt that a transfusion was essential because of a clinical deterioration even if the forearm FOE was $<0.47$. The latter criterion was intended to be a safety mechanism in case of failure of the peripheral FOE to detect clinically significant anaemia, as this measurement had not previously been used for this purpose.

Measurement of FOE by NIRS with partial venous occlusion is a method that has been validated and described elsewhere. ${ }^{710}$ In brief, the monitoring optodes were positioned on the surface of the upper forearm. A short venous occlusion using a blood pressure cuff produced a rise in haemoglobin content of the tissues. $\mathrm{SvO}_{2}$ was calculated by analysing the relative increases in concentration of oxyhaemoglobin and deoxyhaemoglobin during the occlusion." The forearm FOE was calculated from measurements of $\mathrm{Svo}_{2}$ made in this way and $\mathrm{SaO}_{2}$ measured by pulse oximetry. ${ }^{7}$ Measurements were made when infants were at rest, between feeds, and not crying or agitated. When a measurement of FOE was $>0.40$, it was repeated and the two values were then averaged to give the final result.

\section{Transfusion criteria}

Group 1

- Transfused at $\mathrm{Hb}$ of $140 \mathrm{~g} / \mathrm{l}$ if inspired oxygen concentration $>0.35$ or mean airway pressure $>6 \mathrm{~cm} \mathrm{H}_{2} \mathrm{O}$

- Transfused at $\mathrm{Hb}$ of $120 \mathrm{~g} / \mathrm{l}$ if inspired oxygen concentration $<0.35$ or mean airway pressure $<6 \mathrm{~cm} \mathrm{H}_{2} \mathrm{O}$

- Significant apnoeas or bradycardias (more than nine in 12 hours), or if heart rate $>180 / \mathrm{min}$ or respiratory rate $>80$ / min for 24 hours, or if weight gain $<10 \mathrm{~g} /$ day for four days on $418 \mathrm{~kJ} / \mathrm{kg} / \mathrm{day}$.

\section{Group 2}

- Transfused at FOE $>0.47$ or if significant clinical concern by clinicians responsible for the patient.

\section{Blinding}

The nature of the study made it impossible to blind the researchers and the clinical team responsible for the infant's medical care to the research allocation. Forearm FOE measurements were made on all infants in both groups. These results were only available to the researchers and not to the clinical team. Routine full blood counts were made on all infants on a regular basis with similar frequency in the two groups; these were used to guide the need for transfusions in group 1 but not in group 2 . These results were available to both clinical team and researchers.

\section{Treatment protocol for all infants}

Measurements of both $\mathrm{Hb}$ and peripheral FOE were usually made daily for the first week of monitoring, then about four times a week until the infant was 30 weeks postconceptional age, then about twice a week. Peripheral FOE measurements were made each time an infant in either group had a $\mathrm{Hb}$ measurement, and the two groups were sampled routinely for other purposes. The same amount of blood sampling was therefore performed in each group. Full blood counts and other routine tests were carried out according to routine clinical practice and as clinically indicated.

\section{Blood transfusions}

The volume of blood transfused to the infants in both groups was $20 \mathrm{ml} / \mathrm{kg}$ of packed red cells with each transfusion and was not calculated using the Hb before transfusion. All transfused blood was preserved using citrate phosphate. Dedicated units of blood were used for each infant, which were divided into six smaller packs ("Paedipacks") with a total shelf life of 35 days. The number of donors and the number of transfusions were recorded prospectively.

All infants were given either total parenteral nutrition or enteral feeds of breast milk or formula milk. Enteral feeds were increased as tolerated and all infants were given multivitamins ( $0.2 \mathrm{ml} /$ day) and folate supplements (1 $\mathrm{mg} /$ week) from day 14 of life onwards and iron edentate supplements (1 $\mathrm{mg}$ /day) from day 28 onwards.

Oxygen supplementation was given to infants to maintain arterial oxygen saturations between 88 and $95 \%$ for infants of $<32$ weeks gestation and $>94 \%$ for infants of $>32$ weeks gestation..$^{14}$

Infants were discharged home when they were clinically stable, not having apnoeas, and were bottle or breast feeding adequately. 
Table 1 Details of infants before randomisation

\begin{tabular}{lll}
\hline & $\begin{array}{l}\text { NIRS } \\
(\mathrm{n}=37)\end{array}$ & $\begin{array}{l}\text { Conventional } \\
(\mathrm{n}=37)\end{array}$ \\
\hline Birth weight (g) & 1200 & 1136 \\
& $(1004-1373)$ & $(1009-1285)$ \\
Gestational age (weeks) & $29(27-31)$ & $30(27-32)$ \\
Infants less than/equal to $1000 \mathrm{~g}$ & $9(24 \%)$ & $9(24 \%)$ \\
Postnatal age at randomisation (days) & $5(3-8)$ & $5(3-8)$ \\
Hb at randomisation (g/l) & 160 & 155 \\
& $(149-179)$ & $(145-181)$ \\
Transfusions before randomisation & $0(0-1)$ & $0(0-1)$ \\
Ventilated (at any time) & $21(57 \%)$ & $17(46 \%)$ \\
Fraction of inspired oxygen (\%) at & $21(21-23)$ & $21(21-21)$ \\
randomisation & & \\
\hline
\end{tabular}

Results are given as median (range) or number (percentage) NIRS, Near infrared spectroscopy-that is, group assessed using this technique.

\section{Outcome measures}

The primary outcome measure was the number of transfusions received after randomisation. The number of transfusions received before randomisation was recorded but not used in the analysis of the primary outcome. Predetermined secondary outcome measures were rate of weight gain, rate of linear growth (assessed using leg length measurements using knemometry; FORCE Institutes, Copenhagen, Denmark), number of days on the neonatal unit, chronic lung disease (oxygen requirement at 36 weeks postconceptional age), treated retinopathy of prematurity, severe intraventricular haemorrhage (with ventricular dilatation or parenchymal involvement), cystic periventricular leucomalacia, and necrotising enterocolitis requiring surgical intervention.

\section{Sample size}

The sample size was calculated from a survey of transfusions on our own unit over the preceding six months. The mean number of transfusions (range) given to infants less than $1500 \mathrm{~g}$ after the second week of life was $2.8(0-12)$ using the transfusion protocol used for the control group. A previous observational study had shown that about two thirds of infants with symptoms ascribed to anaemia had FOE $<0.47$. $^{7}$ A reduction of two transfusions per infant was felt to be clinically important and required 34 infants in each group to achieve $80 \%$ power using a significance level of $5 \%$.

\section{Statistical and data analysis}

The data were analysed using SPSS for Windows (version 4). All data were analysed on an intention to treat basis. Comparisons between the two groups were made using the Mann-Whitney U test for non-parametric data. Categorical data were analysed using $\chi^{2}$ analysis. FOE is normally distributed, and changes in FOE were analysed by parametric statistics. Hb is not normally distributed and therefore medians and interquartile ranges (IQRs) were used with non-parametric statistics.

\section{RESULTS}

Seventy four infants were randomised, 37 in the NIRS group and 37 in the conventional group.

Table 1 summarises descriptive data for the two groups. There were no clinically important differences between the two groups in terms of birth weight, gestational age, postnatal age at randomisation, $\mathrm{Hb}$ at randomisation, number of transfusions before randomisation, number of infants ventilated, or $\mathrm{FIO}_{2}$.

Two deaths occurred in each group. In the conventional group, one infant died from necrotising enterocolitis and one from sepsis. In the NIRS group, one infant died from bronchopulmonary dysplasia and one from a cardiorespiratory arrest. Fifty six transfusions were given to the 37 infants in the NIRS group compared with 84 to the 37 infants in the conventional group. However, the median number of transfusions per infant was not significantly different between the two groups (table 2). Figure 1 shows the distribution of the number of transfusions. There was also no difference in the median volume of blood given to infants in each group nor the number of donors to which each infant was exposed (table 2). The results from infants with birth weights $\leqslant 1000 \mathrm{~g}$ and $>1000 \mathrm{~g}$ were similar to the group as a whole.

The postnatal age at discharge, weight at discharge, rate of weight gain, and rate of linear growth were not significantly different between the two groups (table 2).

\section{Indications for transfusions}

In the NIRS group, of the 56 transfusions that were given, 18 $(32 \%)$ were because of high FOE. Thirty seven $(66 \%)$ were given for clinical reasons before the infants' FOE had reached the treatment value; $23(41 \%)$ of these were given because of symptoms ascribed to anaemia, and 10 were given because of low $\mathrm{Hb}$. One baby in the NIRS group was transfused according to the wrong protocol.

\begin{tabular}{|c|c|c|c|}
\hline & NIRS $(n=37)$ & Conventional $(n=37)$ & $\mathrm{p}$ Value \\
\hline Total number of transfusions & 56 & 84 & \\
\hline Number of transfusions per infant & $\begin{array}{l}1(0-2) \\
\text { range } 0-8\end{array}$ & $\begin{array}{l}1(0-3) \\
\text { range 0-14 }\end{array}$ & 0.64 \\
\hline Number of donor exposures & $1(0-2)$ & $1(0-2)$ & 0.68 \\
\hline Volume of blood per infant (ml) & $36(0-71)$ & $37(0-84)$ & 0.81 \\
\hline Infants receiving no transfusions & $13(35 \%)$ & $15(41 \%)$ & 1.0 \\
\hline Infants receiving 1 or less transfusions & $23(62 \%)$ & $20(54 \%)$ & 0.64 \\
\hline Infants receiving 2 or less transfusions & $29(78 \%)$ & $25(68 \%)$ & 0.43 \\
\hline Infants receiving 3 or less transfusions & $34(92 \%)$ & $29(78 \%)$ & 0.19 \\
\hline Weight gain (g/week) & $126(111-153)$ & $128(113-144)$ & 0.99 \\
\hline Length gain (mm/week) & $2.8(2.5-3.2)$ & $3.2(2.6-3.4)$ & 0.25 \\
\hline Weight at discharge $(\mathrm{g})$ & $2214(1940-2788)$ & $2064(1874-2360)$ & 0.19 \\
\hline Postmenstrual age at discharge (weeks) & $37(36-39)$ & $37(36-39)$ & 0.72 \\
\hline Length of stay (days) & $58(35-84)$ & $49(41-70)$ & 0.46 \\
\hline Deaths & $2(5 \%)$ & $2(5 \%)$ & 1.0 \\
\hline CLD at 36 weeks & $13(35 \%)$ & $12(32 \%)$ & 0.81 \\
\hline Necrotising enterocolitis & 1 & 1 & 1 \\
\hline Treated retinopathy & 0 & 0 & 1 \\
\hline
\end{tabular}




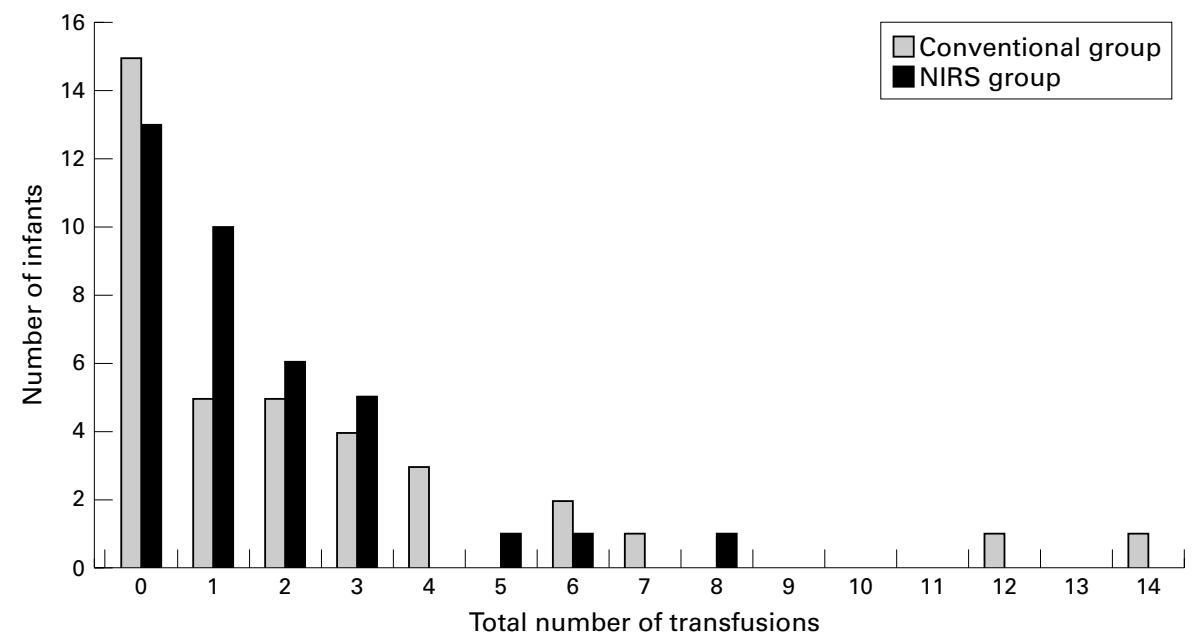

Figure 1 Distribution of number of transfusions per infant in the two randomised groups. NIRS, Near infrared spectroscopy.

In the conventional group, $64(76 \%)$ of the transfusions were given because of low $\mathrm{Hb}$ alone, and 17 (20\%) were given because of symptoms ascribed to anaemia as defined in the protocol. Two ( $2 \%$ ) were given because of acute haemorrhage and one $(1 \%)$ was given before surgery. Table 3 shows the reasons for transfusion in the two groups. More infants were transfused for symptoms attributed to anaemia (in most cases apnoeas and bradycardias) in the NIRS group than in the conventional group $(p=0.01)$.

Table 4 shows the changes in FOE and Hb after transfusion. Infants transfused for symptoms ascribed to anaemia in the conventional group had a mean (SD) FOE of 0.415 (0.07). This was significantly higher than that in the corresponding NIRS group $(0.355(0.05))(\mathrm{p}=0.04)$. The median (IQR) Hb was

\begin{tabular}{lll}
\hline Table 3 Indications for transfusions in the two groups \\
\hline Indication for transfusion & $\begin{array}{c}\text { Number of } \\
\text { transfusions in } \\
\text { NIRS group }\end{array}$ & $\begin{array}{l}\text { Number of } \\
\text { transfusions in } \\
\text { conventional group }\end{array}$ \\
\hline FOE $>0.470$ & 18 & 0 \\
Low Hb (according to protocol) & 0 & 64 \\
Low Hb alone $(<80 \mathrm{~g} / \mathrm{ll})$ & 2 & 0 \\
Transfusion given according to wrong protocol & 1 & 0 \\
Acute clinical deterioration including apnoeas and/or desaturations & 22 & 17 \\
Acute haemorrhage & 2 & 2 \\
Clinical concern because of high dependency and low Hb & 8 & 0 \\
No indication recorded & 1 & 0 \\
Breathless on feeding & 1 & 0 \\
Before surgery & 1 & 1 \\
Total & 56 & 84 \\
\hline NIRS, Near infrared spectroscopy; FOE, fractional oxygen extraction; Hb, haemoglobin concentration. \\
\hline
\end{tabular}

Table 4 Changes in frational oxygen extraction (FOE) and haemoglobin concentration $(\mathrm{Hb})$ with transfusion

\begin{tabular}{|c|c|c|c|c|c|}
\hline \multirow[b]{2}{*}{ Indication for transfusion } & \multicolumn{2}{|l|}{ FOE } & \multirow{2}{*}{$\begin{array}{l}\text { Significance } \\
\text { of changes in } \\
\text { FOE }\end{array}$} & \multicolumn{2}{|l|}{$\mathrm{Hb}(\mathrm{g} / \mathrm{l})$} \\
\hline & Before transfusion & After transfusion & & Before transfusion & After transfusion \\
\hline \multicolumn{6}{|l|}{ NIRS } \\
\hline High FOE & $\begin{array}{l}0.508(0.06) \\
\text { Mean change }(95 \% \mathrm{Cl})\end{array}$ & $\begin{array}{l}0.328(0.06) \\
0.180(0.136 \text { to } 0.225)\end{array}$ & $p<0.001$ & $\begin{array}{l}88(75-97) \\
\text { Mean change }(95 \% \mathrm{Cl})\end{array}$ & $\begin{array}{l}130(125-169) \\
-46(-52 \text { to }-40)\end{array}$ \\
\hline Low $\mathrm{Hb}$, high $\mathrm{FlO}_{2}$ & $\begin{array}{l}0.340(0.06) \\
\text { Mean change }(95 \% \mathrm{Cl})\end{array}$ & $\begin{array}{l}0.298(0.06) \\
0.052(0.021 \text { to } 0.084)\end{array}$ & $p=0.005$ & $\begin{array}{l}98(80-111) \\
\text { Mean change }(95 \% \mathrm{Cl})\end{array}$ & $\begin{array}{l}145(119-148) \\
-43(-50 \text { to }-36)\end{array}$ \\
\hline Symptoms & $\begin{array}{l}0.355(0.05) \\
\text { Mean change }(95 \% \mathrm{Cl})\end{array}$ & $\begin{array}{l}0.333(0.04) \\
0.031(0.0037 \text { to } 0.066)\end{array}$ & $p=0.07$ & $\begin{array}{l}87(76-105) \\
\text { Mean change }(95 \% \mathrm{Cl})\end{array}$ & $\begin{array}{l}130(125-169) \\
-54(-73 \text { to }-35)\end{array}$ \\
\hline \multicolumn{6}{|l|}{ Conventional } \\
\hline Low $\mathrm{Hb}$ & $\begin{array}{l}0.351(0.05) \\
\text { Mean change }(95 \% \mathrm{Cl})\end{array}$ & $\begin{array}{l}0.299(0.06) \\
0.053(0.032 \text { to } 0.073)\end{array}$ & $p=0.01$ & $\begin{array}{l}121(108-130) \\
\text { Mean change }(95 \% \mathrm{CI})\end{array}$ & $\begin{array}{l}155(146-168) \\
-39(-43 \text { to }-34)\end{array}$ \\
\hline Symptoms & $\begin{array}{l}0.415(0.07) \\
\text { Mean change }(95 \% \mathrm{Cl})\end{array}$ & $\begin{array}{l}0.332(0.08) \\
0.082(0.018 \text { to } 0.147)\end{array}$ & $p=0.02$ & $\begin{array}{l}102(92-114) \\
\text { Mean change (95\% Cl) }\end{array}$ & $\begin{array}{l}141(134-160) \\
-43(-52 \text { to }-33)\end{array}$ \\
\hline
\end{tabular}

FOE values are given as mean (SD); $\mathrm{Hb}$ values are given as median (interquartile range).

NIRS, near infrared spectroscopy. 
Table 5 Fractional oxygen extraction (FOE) and haemoglobin concentration $(\mathrm{Hb})$ during the study

\begin{tabular}{llll}
\hline & NIRS & Conventional & $\mathrm{p}$ Value \\
\hline Hb at randomisation $(\mathrm{g} / \mathrm{l})$ & $160(149-179)$ & $155(145-181)$ & 0.76 \\
Lowest $\mathrm{Hb}(\mathrm{g} / \mathrm{l})$ & $85(77-120)$ & $96(88-118)$ & 0.009 \\
$\mathrm{Hb}$ at $1^{\text {st }}$ transfusion $(\mathrm{g} / \mathrm{l})$ & $92(74-110)$ & $116(104-120)$ & 0.0004 \\
$\mathrm{Hb}$ at discharge $(\mathrm{g} / \mathrm{l})$ & $104(91-130)$ & $120(95-143)$ & 0.15 \\
$\mathrm{FOE}$ at randomisation & $0.334(0.08)$ & $0.331(0.06)$ & 0.86 \\
Highest FOE & $0.443(0.06)$ & $0.444(0.06)$ & 0.96 \\
FOE at 1st transfusion & $0.425(0.08)$ & $0.354(0.09)$ & 0.01 \\
FOE at discharge & $0.346(0.07)$ & $0.388(0.07)$ & 0.01 \\
\hline
\end{tabular}

$\mathrm{FOE}$ values are given as mean (SD); $\mathrm{Hb}$ values are given as median (interquartile range).

NIRS, Near infrared spectroscopy.

$102(92-114) \mathrm{g} / \mathrm{l}$ in the conventional group with symptoms $v$ 87 (76-105) g/l in the NIRS group with symptoms $(p=0.08)$.

In the NIRS group, transfusions were given for high FOE, for symptoms thought by the treating clinician to be due to anaemia, or because of a low $\mathrm{Hb}$ in an infant who had a high clinical dependency (usually a high $\mathrm{FIO}_{2}$ ). There were no differences in the median $\mathrm{Hb}$ at the time of transfusion in these three groups (table 4).

Infants in the conventional group received their first transfusion earlier than those in the NIRS group. They were first transfused at a median (IQR) age of 12 (6-21) days, whereas infants in the NIRS group received their first transfusion at a median (IQR) age of $25(13-40)$ days $(p=0.006)$.

\section{Haemoglobin concentrations}

In table 5 the $\mathrm{Hb}$ at different time points during the study are compared. There was no difference in $\mathrm{Hb}$ at randomisation, but the NIRS group had a lower median $\mathrm{Hb}$ during the study and a lower $\mathrm{Hb}$ at the time of the first transfusion. There was no difference in $\mathrm{Hb}$ between the groups at the time of discharge.

The median (IQR) number of days spent with $\mathrm{Hb}<100 \mathrm{~g} / \mathrm{l}$ in the control group was $1(0-8)$, which was significantly lower than the number of days $<100 \mathrm{~g} / \mathrm{l}$ in the NIRS group ( 8 $(0-20)(p=0.01)$.

\section{FOE results}

Table 5 also shows a comparison of the FOE results from the two groups. There was no difference in FOE at the time of randomisation, but it was lower in the conventional group at the time of the first transfusion and higher in this group at the time of discharge.

The median (IQR) number of days spent with FOE $>0.40$ in the control group was $5(0.5-11)$, which was not significantly different from the number of days with FOE $>0.4$ in the NIRS group $(6(1-10))(p=0.57)$

\section{DISCUSSION}

Fewer transfusions were given to the 37 infants in the NIRS group than to those in the group transfused according to conventional criteria ( $56 v 84$ ), but there was no difference in the average number of transfusions given.

There are several possible reasons for the failure to show a significant difference in the number of transfusions received by infants in the two groups. Firstly, many transfusions were given to infants in the NIRS group who did not have an FOE of $>0.470$. These infants were transfused on the basis of a low $\mathrm{Hb}$ alone or because of non-specific symptoms, which may often have been due to clinical reasons other than anaemia, such as infection. In this study the infants who were transfused because of symptoms attributed to anaemia in the conventional group had a higher FOE than infants without symptoms in the same group. However, in the NIRS group, infants transfused by clinicians because of symptoms ascribed to anaemia had a mean FOE of $0.346(0.06)$, which is similar to measurements in non-anaemic control infants. This suggests that infants classified as having symptoms in the NIRS group did not have decreased oxygen delivery despite a low $\mathrm{Hb}$. In the NIRS group, when an infant's FOE was high because of significant anaemia they were transfused, and this is likely to have happened before symptoms developed in these infants so that symptoms directly attributable to anaemia should have been less likely.

More transfusions in the NIRS group were given because of symptoms ascribed to anaemia. However, it is not known whether all of these symptoms were caused by the anaemia or were due to other factors and were ascribed to the anaemia because the haemoglobin concentration was low. The fact that there was no difference in outcome in infants in the two groups is reassuring as adverse effects would have been noted if they were being undertransfused. Infants in this group did not spend a longer period with high FOE values.

The rationale for the use of a peripheral FOE value of 0.47 to guide transfusions is that peripheral FOE measurements would correctly identify infants with symptomatic anaemia. ${ }^{7}$ A possible explanation for the failure of the study to show the expected differences is a failure of peripheral FOE to identify infants with significant anaemia. The limit of FOE at which transfusion was undertaken was derived from a previously established reference range, but may have been too high, so that some infants with clinically significant anaemia may not have been correctly identified. This would explain the large number of infants transfused because of clinical concern. It is possible that peripheral FOE varies as the result of measurement error and physiological changes so that a single measurement of FOE is not sufficiently sensitive to identify all infants with clinically significant anaemia.

Another possible explanation for the higher than expected number of transfusions given to the NIRS group is that the peripheral FOE measurements were made when the infants were at rest and not stressed. Although oxygen delivery to tissues may be adequate most of the time, it is possible that tissue oxygen supply becomes compromised when infants are stressed or active and demands for oxygen increase-for instance, during feeding. If this is the case, measuring infants at rest may not be ideal, and the method may be more sensitive if measurements are made when infants are stressed. This is an area for further investigation.

In this study, despite the reduction in the total number of transfusions and the significantly lower median $\mathrm{Hb}$ in the NIRS group, there was no apparent effect on growth, in terms of weight gain, linear growth, or length of stay. The evidence for anaemia causing poor weight gain and growth is lacking, although it is often given as an indication for transfusion. Four previous studies have addressed this. ${ }^{15-18}$ None of them give information about the energy intake of the infants considered, and the results are conflicting. ${ }^{2}$ As so many factors affect 
growth, it is perhaps not that surprising that anaemia may have only a small role, if any.

The infants in the NIRS group were older when first transfused than infants in the conventional group. Transfusions in this group may have been delayed rather than avoided. An alternative explanation may be that, because of changes in the concentration of $\mathrm{HbF}$, measurement of FOE is better at detecting the need for transfusion in infants with a greater postnatal age. Delaying transfusion may be beneficial as long as infants are not physiologically compromised because stimulation of erythropoiesis may reduce further transfusion need. The transfusion protocol in the conventional group was a standard one, but it allowed many transfusions early in their course to some infants, and these may have been unnecessary.

There are many potential advantages to limiting the number of blood transfusions given to preterm infants. Not only are infants exposed to fewer donors with a resultant reduced risk of infective complications, but the risk of complications such as chronic lung disease and retinopathy of prematurity may also be reduced. ${ }^{19-23}$ The mechanism of this relation has been said to be due to the development of oxygen free radicals catalysed by the free iron in blood transfusions. ${ }^{24}$ This study was too small to show differences in the rates of chronic lung disease or retinopathy of prematurity between the two groups.

The time at which infants $<1000 \mathrm{~g}$ receive most transfusions is during the first 2 weeks of life. To show a difference, it would be necessary to reduce the number of transfusions during this period, a time when they are also clinically unstable. We feel that further work is needed before this technique can be applied to such critically ill infants.

Although this study has failed to show a significant reduction in the number of transfusions, fewer transfusions were given to the NIRS group. If the results from this pilot studythat is, a reduction in the mean number of transfusions from 2.3 to 1.5 -are used to calculate a sample size, 251 infants would be required in each group to show a similar difference (power $80 \%$, significance 5\%). Refinements or improvements in the technique may also be possible to improve the sensitivity of the method to detect clinically significant anaemia.

Despite the fact that blood transfusions are part of routine care of the preterm infant, there are few trials that help to guide practice. The only published randomised controlled trial of two different transfusion regimens showed no advantage in keeping $\mathrm{Hb}$ above $100 \mathrm{~g} / \mathrm{l} .{ }^{15}$ The infants in that study were of similar birth weight and gestational age to the infants in our study, but the number of blood transfusions given was not reported as it was not a primary outcome measure. Another possible way of improving the technique is to combine measurements of peripheral FOE with other measures of the adequacy of tissue oxygenation to produce a set of criteria for giving transfusions in preterm infants. Further refinements of the technique may enable better targeting of transfusions resulting in fewer of them. Studies to produce new evidence based guidelines for blood transfusions are urgently required.

\section{Authors' affiliations}

S P Wardle, R Garr, C W Yoxall, A M Weindling, Neonatal Unit, Liverpool Women's Hospital, Liverpool, UK

\section{REFERENCES}

1 Strauss RG. Red blood cell transfusion practices in the neonate. Clin Perinatol 1995;22:641-55.

2 Ramasethu J, Luban NLC. Red blood cell transfusions in the newborn. Semin Neonatol 1999;4:5-16.

3 Holland BM, Jones JG, Wardrop CA. Lessons from the anemia of prematurity. Hematol Oncol Clin North Am 1987;1:355-66.

4 Alverson DC, Isken VH, Cohen RS. Effect of booster blood transfusions on oxygen utilization in infants with bronchopulmonary dysplasia. Effect of booster blood transfusions on oxygen utilization in infants with bronchopulmonary dysplasia. J Pediatr 1988;1 13:722-6.

5 Wardrop CA, Holland B, Veale KE, et al. Nonphysiological anaemia of prematurity. Arch Dis Child 1978;53:855-60.

6 Alverson DC. The physiologic impact of anemia in the neonate. Clin Perinatol 1995;22:609-25.

7 Wardle SP, Crawley E, Yoxall CW, et al. Peripheral oxygenation and anaemia in preterm babies. Pediatr Res 1998;44:125-31.

8 Lister G, Moreau G, Moss M, et al. Effects of alterations of oxygen transport on the neonate. Semin Perinatol 1984;8:192-204.

9 Yoxall CW, Weindling AM. The measurement of peripheral venous oxyhemoglobin saturation in newborn infants by near infrared spectroscopy with venous occlusion. Pediatr Res 1996;39:1 103-6.

10 Yoxall CW, Weindling AM. Measurement of venous oxyhaemoglobin saturation in the adult human forearm by near infrared spectroscopy with venous occlusion. Med Biol Eng Comput 1997;35:331-6.

11 Jones JG, Holland BM, Veale KE, et al. 'Available oxygen', a realistic expression of the availability of the blood to supply oxygen to the tissues. Scand J Haematol 1979;22:77-82.

12 Jones JG, Holland BM, Hudson IR, et al. Total circulating red cells versus haematocrit as the primary descriptor of oxygen transport by the blood. Br J Haematol 1990;76:288-94.

13 Shannon KM, Keith JF, Mentzer WC, et al. Recombinant human erythropoietin stimulates erythropoiesis and reduces erythrocyte transfusions in very low birth weight preterm infants. Pediatrics 1995; $95: 1-8$

14 Cochran DP, Shaw NJ. The use of pulse oximetry in the prevention of hyperoxaemia in preterm infants. Eur J Pediatr 1995;154:222-4.

15 Blank JP, Sheagren TG, Vajaria J, et al. The role of RBC transfusion in the premature infant. American Journal of Diseases in Children 1984; 138:831-3

16 Meyer J, Sive A, Jacobs P. Empiric red cell transfusion in asymptomatic preterm infants. Acta Paediatr 1993:82:30-4.

17 Lachance C, Chessex P, Fouron JC, et al. Myocardial, erythropoietic, and metabolic adaptations to anemia of prematurity. $J$ Pediatr 1994; 125:278-82

18 Stockman JA, Clark DA. Weight gain: a response to transfusion in selected preterm infants. American Journal of Diseases in Children 1984;138:828-830

19 Clark J, Gibbs JAH, Maniello R. Blood transfusion: a possible risk factor in retrolental fibroplasia. Acta Paediatr Scand 1981;70:535-9.

20 Cooke RWI, Clark D, Hickey-Dwyer M, et al. The apparent role of blood transfusions in the development of retinopathy of prematurity. Eur $J$ Pediatr 1993;152:833-6.

21 Sacks LM, Delivoria Papadopoulos M, Anday EK, et al. Retrolental fibroplasia and blood transfusion in very low-birth-weight infants. Pediatrics 1981;68:770-4.

22 Shohat $M$, Reisner SH, Krikler R, et al. Retinopathy of prematurity: incidence and risk factors. Pediatrics 2000;72:159-63

23 Griffiths G, Lall R, Chatfield S, et al. Randomised controlled double blind study of recombinant erythropoeitin in the prevention of chronic lung disease. Arch Dis Child Fetal Neonatal Ed 1997;76:190-2.

24 Sullivan JL. Iron, plasma antioxidants, and the 'oxygen radical disease of prematurity'. American Journal of Diseases in Children 1988;142:1341-4 